\title{
CAR SECURITY SYSTEM BASED ON RASPBERRY PI
}

\author{
MOHANAD ABDULHAMID ${ }^{1}$, ACHIKI MATONGO $^{2}$ \\ ${ }^{1}$ AL-Hikma University, Iraq, ${ }^{2}$ University of Nairobi, Kenya \\ E-mail: moh1hamid@yahoo.com, researcher12018@yahoo.com
}

\begin{abstract}
Statistics show that there is an increase in production of cars every year worldwide. Similarly, there is an increase in car theft attempts and carjacking cases worldwide. This paper is meant to increase the user's situational awareness. The user is able to check for surrounding of the vehicle and judge for himself/ herself before approaching the vehicle. Raspberry Pi is used as the controller in this paper. A Universal Serial Bus (USB) webcam plugged into the Raspberry Pi enables the user to view the images captured in real time by use of an application installed on the user's smartphone.
\end{abstract}

Keywords: Car security system, Raspberry Pi, design.

\section{INTRODUCTION}

Security and safety of a person property are some of the basic rights that a human being should enjoy. However owing to the few elements in the society, security is not necessarily guaranteed specifically to a person's car. Hence there is need to design a simple system that allows for the user to monitor the car and check for intruders before the user approaches the car. In addition, the system alerts the user on threats in case an intruder breaks into the car $[1,2]$.

State of the art car security systems are available in the market. For example, Viper 3303 or Python 991 offer the user a real time check on the security of the car. The cost of installation of such systems is a challenge. This paper aims to find a cheaper alternative to the car security system [3].

Statistics indicate that cases of carjacking and car theft are on the rise yearly. Due to these reason, this paper is aimed at increasing the user's awareness such that he/she can view the surrounding before approaching the car [4].

Car security system is meant to increase the awareness of the user with regards to his/her car in their absence. The system uses a camera that captures images and streams them on a webpage for the user to view and access the situation before approaching the car. This enhances users decision with reference to security $[5,6]$.

The scope of the paper involves design and implementation of a Raspberry Pi based car security system. Camera, sensors output and commands sent by the user serve as the input to the controller and the output is a text message or an image.

\section{DESIGN AND IMPLEMENTATION}

\subsection{Hardware design}

The hardware design contains the following modules:

- Raspberry Pi;

- USB webcam;

- User interface;

- Power supply.

The block diagram of the various modules is shown in Figure 1.

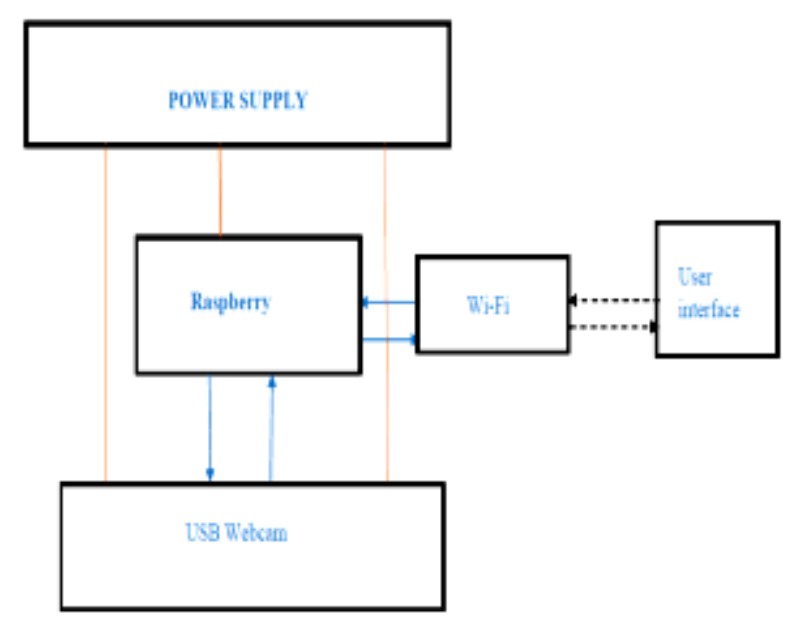

Figure 1. Block diagram of car security system

\subsubsection{Raspberry Pi}

Raspberry Pi is chosen as the control unit. The USB webcam is mounted on one of the USB ports on the Raspberry Pi board. A software on the Raspberry Pi enables the user to stream the images in real time on a web page whenever he/she wishes to check the surrounding for intruders. Connection to the Raspberry Pi control unit is via Wi-Fi. That is to say both the Raspberry Pi and the user's smartphone are connected via a local network, in this particular case, it is a Wi-Fi hotspot. This method is used since it offers the user adequate security in terms of access to the images.

\subsubsection{Camera}

A webcam is a video camera that has the capability to stream pictures or videos to a computer or to a remote computer connected in a network. This is the major point 
of our paper. User situational awareness is increased by means of a surveillance camera that can be activated remotely at the request of the user. The webcam used in this paper is a low cost USB webcam that is connected directly to the USB ports of the Raspberry Pi which serves as the control unit. Using a software installed on the Raspberry Pi and configured specifically to command the webcam to take and stream pictures, the webcam captures photos of any person or moving object which can then be accessed through another device remotely. Otherwise, the pictures can be sent to the user's email account or dropbox account depending on the user's preference.

\subsubsection{User interface}

User interface refers to the mode of interaction between a computer or machine and a human user. The main aim of a user interface is to allow for effective communication between the computer and the user. In this paper, the user interface is an application stored in the user's smartphone that allows the user to turn on the Raspberry Pi controller remotely and also activate the camera to check for intruders if any and send the images captured to the user's email account or dropbox account.

\subsubsection{Power supply}

Power supply refers to the system that supplies the Raspberry Pi with power for the normal functioning and execution of tasks as directed by the user. Raspberry Pi requires $5 \mathrm{~V}, 2 \mathrm{~A}$ for its normal usage. It's also important to note that Raspberry Pi can be powered in two different ways. The first method simply involves using a USB cable and a standard smartphone charger rated at $5 \mathrm{~V}, 2 \mathrm{~A}$. The second method involves using pin $2(5 \mathrm{~V})$ and pin 6 (Ground) and connect them to power supply. In this case, a $5 \mathrm{~V}$ voltage regulator is designed to meet the power requirements of the system. For a car, the design shown in Figure 2 is appropriate.

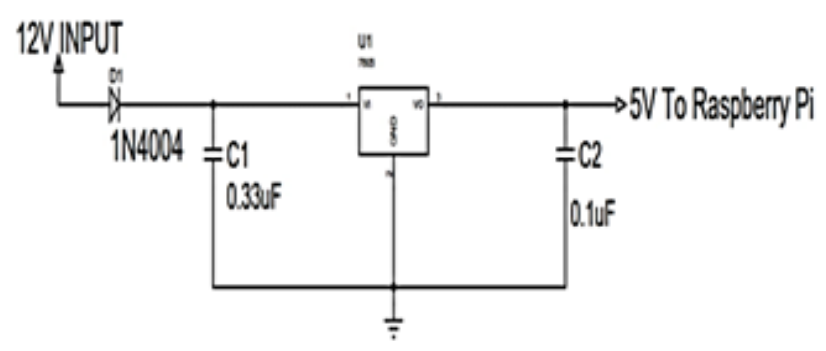

Figure 2.5V power supply

\subsection{Software design}

To monitor the surrounding, a program is used to enable the user stream the images in real time by using a browser such as Mozilla Firefox and a media player such as Video LAN Client (VLC) media player. A video compression format that compresses JPEG images is used. The format used is called M-JPEG (Motion-JPEG). M-JPEG is mainly used because it uses hardware compression of webcams hence reduces Central Processing Unit (CPU) cycles of the server. This makes it light and hence less demanding to the CPU power when compressing video frames. This method also uses the standard streaming HTTP protocol for communication in the World Wide Web. It's also important to note that M-JPEG streamer doesn't require additional plugins to stream the images hence making it simple to use for the human end user.

\section{RESULTS}

The images in Figure 3 show how the various components are connected together to form the system.
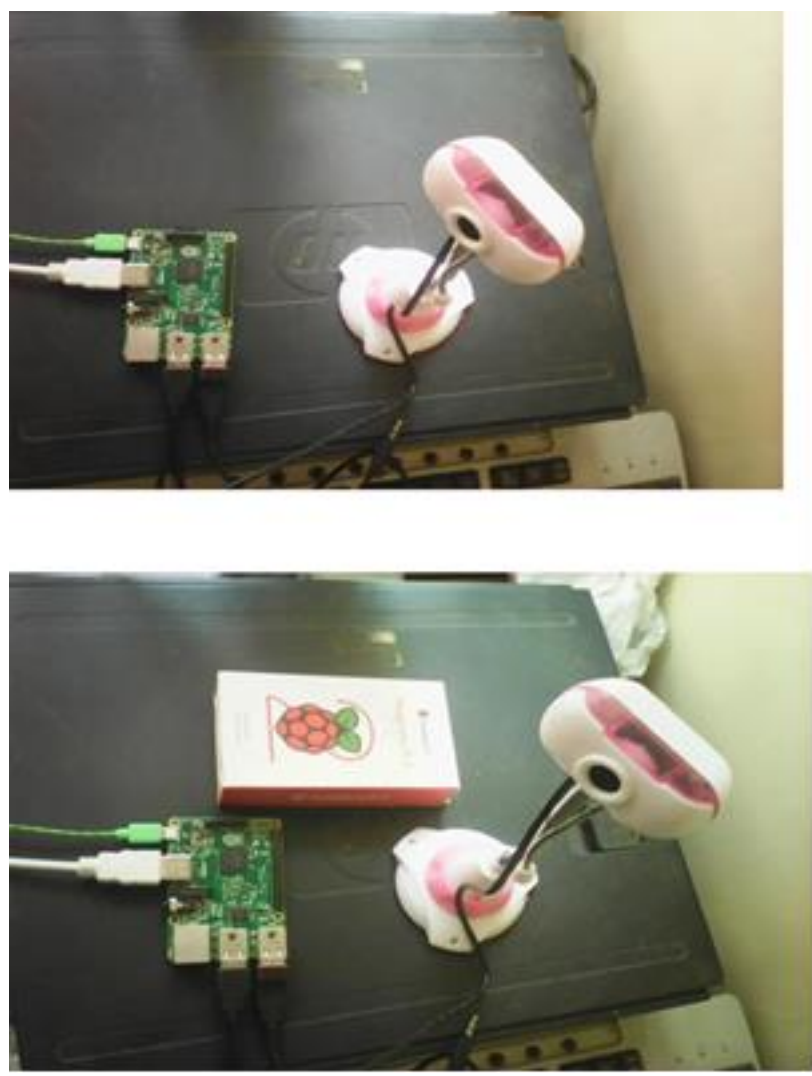

Figure 3. Images of various components

The images shown in Figure 4 are captured by the webcam connected on the Raspberry Pi. The images are screenshots of the live stream from a webpage. Mozilla Firefox is the application used to stream the images. 

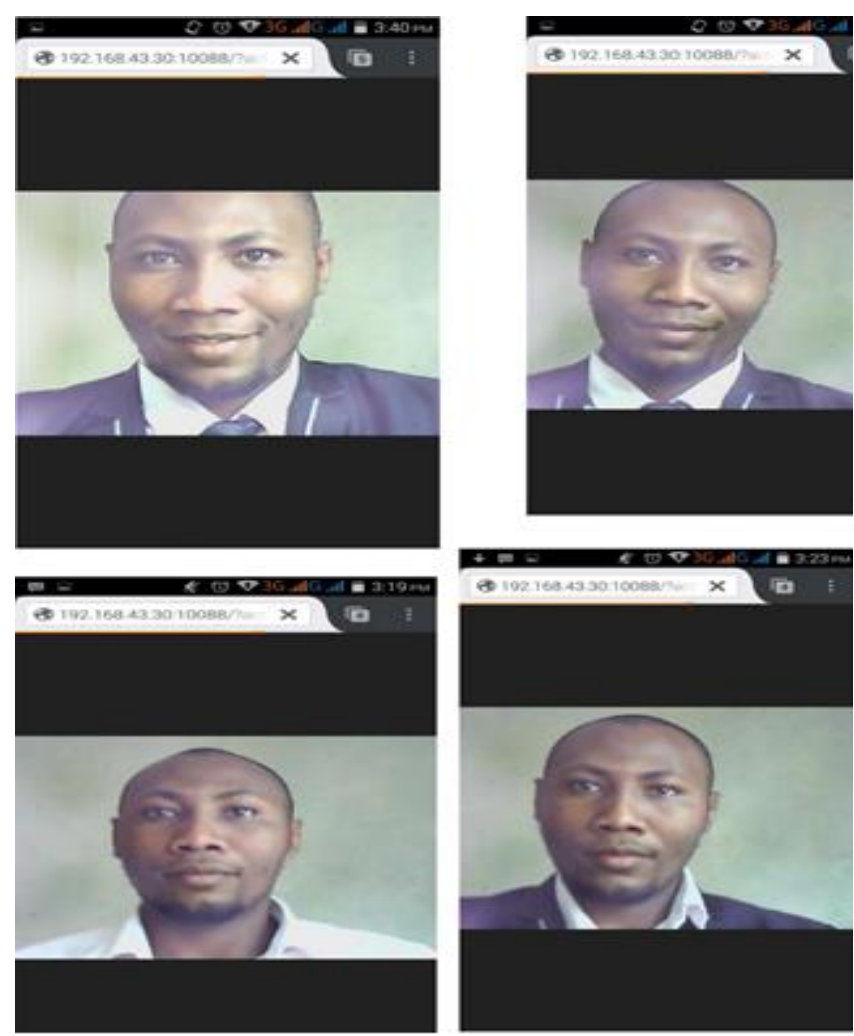

Figure 4. Images of results

\section{CONCLUSION}

A Raspberry Pi based car security system was designed and implemented. User can be able to stream live images using a browser such as Mozilla Firefox. The user is only able to access the images when they are connected in the same local network. A static IP address was used to stream the images in real time on a webpage in Mozilla Firefox. This is because it is assumed that the user doesn't have continues access to a laptop hence unable to search for the IP address. With a static IP address, anyone having access to that IP address can view or stream the images if they know their way around it. Hence it is therefore recommended that future security system should include a software that prompts the user to enter their password in order to have access to the images or whenever they want to stream the images on a webpage.

\section{REFERENCES}

[1] N. Hashim, and M. Halim, "Vehicle security system using Zigbee", International Journal of Scientific and Research Publications, Vol.3, Issue 9, pp. 1-6, 2013.

[2] H. Han, and H. Tun, "Advanced car security system using GSM", International Journal of Scientific and Research Publications, Vol.4, Issue 5, pp. 1-5, 2014.

[3] U. Barua, M. Alamgir, and M. Ali, "MMS based car security system", International Conference on Mechanical, Industrial and Materials Engineering, Bangladesh, 2015.

[4] M. Ahmed, "A review on advanced vehicle security system with theft control and ambulance rescue by intelligent traffic light system", International Journal of Innovation Research in Technology, Vol.3, Issue 3, 2016.

[5] K. Mawonde, and B. Isong, "A survey on vehicle security systems: approaches and technologies", The 44th Annual Conference of the IEEE Industrial Electronics Society, USA, 2018.

[6] S. Rana1, R. Mewari, and L. Nautiyal, "Anti-theft security system for vehicles", International Journal of Engineering \& Technology, Vol.7, No.4, PP.42-46, 2018. 